EXTBNDED BMISSION SOURCES OBSERVED VIA TWO-PROTUN CORRELATIONS*

T. C. Awes, R. L. Ferguson, F. E. Obenshain, F, Plasil, and G. R. Young Oak Ridge National Laboratory, Oak Ridge, Tennessee 37831

\title{
S. Prate $\dagger$
}

CONF-8806117--6

Department of Physics, University of Tennessee Knoxville, Tennessee 37966-1200

Z. Chen, C. K. Gelbke, W. G. Lynch, J. Pochodzalla, $\$$ and H. M. Xu National Superconducting Cyclotron Laboratory Michlgan State University, East Lansing, Michigan 48824-1321

Two-proton correlations were measured as a function of the total energy and relative momentum of the protons. The correlation is analyzed for different orlentations of the relative momentum, which allows information on the size and lifetime of the emission source to be extracted. The most energetic particles are emitted from a short-lived source of compound nucleus dimensions while the lower energy protons appear to be emitted from a source considerably larger than the compound nucleus.

Two-particle correlations between light particles emitted in heavyIon reactions may be used to extract information on the spatial extent and time development of the emission source. Such measurements have provided evidence for the formation and decay of localized regions of high excitation. ${ }^{-5}$ An interesting aspect of these measurements is the observation that the less energetic particles are emitted from sources of larger apparent dimensions. This has been interpreted as an indication that the lower energy particles are emitted at a later, more equilibrated stage of the reaction.6,7 As originally pointed out by Koonin, 8 the dependence of the two-particle correlation on the direchip? of the relative momentum, with respect to the direction of emission, 
provide information on the source lifetime and shape. Although spatial and temporal effects are not strictly distinguishable, a long-lived spherical emission scurce will have a characterlstically prolate appearance, elongated in the direction of emission. 7,8

The experiment was performed at the Holifield Heavy Ion Research Facllity in Oak Ridge. A natural Ag target of $9.9 \mathrm{mg} / \mathrm{cm}^{2}$ areal density was bombarded by $\mathrm{a}^{32} \mathrm{~s}$ beam of $22.3-\mathrm{MeV} /$ nucleon Incident energy. Protons were detected in a closely packed hexagonal array of $13 \Delta \mathrm{E}-\mathrm{E}$ telescopes. Each telescope consisted of a $400-\mu \mathrm{m}-t h 1 \mathrm{ck}$ Si detector and a 10-cm-thick Nal detector, and each subtended a solid angle of $0.67 \mathrm{msr}$ with an angular separation of $4.1^{\circ}$ between adjacent telescopes. The hodoscope was centered at a laboratory angle of $30^{\circ}$. The energy callbration is accurace to within 3\%. An energy threshold of $12 \mathrm{MeV}$ has been used in the off-line analysis of the proton data present- here. The correlation data have been corrected for random $c:$ incidences, which were always at a level of less than $10 \%$.

The correlation function $R(\vec{K}, \vec{q})$ is extracted from the ratio of the coincidence yield $Y_{12}\left(\vec{p}_{1}, \vec{p}_{2}\right)$ to the singles yields $Y_{1}\left(\vec{p}_{1}\right)$ and $Y_{2}\left(\vec{p}_{2}\right)$ of particles 1 and 2 according to:

$$
\left[\mathrm{Y}_{12}\left(\overrightarrow{\mathrm{p}}_{1}, \overrightarrow{\mathrm{p}}_{2}\right)=\mathrm{C}_{12}[1+\mathrm{R}(\overrightarrow{\mathrm{K}}, \overrightarrow{\mathrm{q}})]\left[\mathrm{Y}_{1}\left(\overrightarrow{\mathrm{p}}_{1}\right) \mathrm{Y}_{2}\left(\overrightarrow{\mathrm{p}}_{2}\right)\right.\right.
$$

where $\overrightarrow{\mathrm{P}}_{1}$ and $\overrightarrow{\mathrm{P}}_{2}$ are the momenta of the two protons and $\mathrm{k}$ and $\mathrm{q}$ are the total and relative momenta of the pair, respectively. The single overall normalization constant, $C_{12}$, was chosen such that after summing Eq. (1) over all $|\vec{k}|$ and directions, $\langle R(|\vec{q}|)\rangle=0$ in the interval $52<|\vec{q}|<80$ $\mathrm{MeV} / \mathrm{c}$. For each gating conditton, the sums on both sides of Eq. (l) were extended over all detector and proton energy comblnations corresponding to the given bins of $\stackrel{t}{q}$. 
Examples of the measured directional dependence of the correlation functions are shown in Fig. I for the ${ }^{32} \mathrm{~S}+\mathrm{Ag}$ reaction for gates on the total kinetic energy of the emitted pair of $E_{p p}=60-70$ and $90-100 \mathrm{MeV}$. For each energy gate the correlation is shown for relative momenta either parallel $\left({ }_{\text {rel }}=0^{\circ} \pm 30^{\circ}\right.$, or $\left.180^{\circ} \pm 30^{\circ}\right)$ or transverse $\left(\theta_{\text {rel }}=90^{\circ} \pm\right.$ $30^{\circ}$ ) to the direction of the emitted proton pair. Within statistics, no difference was observed between transverse directions in and out of the reaction plane; therefore, all transverse directions have been summed together. The finite size of the detector hodoscope introduces cutoffs in the relative momentum, which are clearly seen in Fig. l. For relative momenta in the longitudinal direction, the minimum angle between adjacent detectors introduces a cutoff at small q. Similarly, the maximum angle between detectors introduces a cutoff at large $q$ for relative momenta transverse to the direction of emisston. The location of these cutoffs has been verified by a Monte Carlo calculation which included the geometrical acceptance and energy and angular resolution of the hodoscope.

Also shown in Fig. 1 are theoretical results calculated for nonspherical gaussian-shaped sources of longitudinal and transverse "radil" $\mathbf{R}_{\mathrm{L}}$ and $\mathbf{R}_{\mathrm{T}}$.

$$
f_{K}(\vec{r}, t+\infty)=f_{0} \exp -\frac{x^{2}+y^{2}}{R_{T}^{2}}+\frac{z^{2}}{R_{L}^{2}}
$$

The relative wavefunctions were obtained by solving Schroedinger's equation with the Reld soft core futential for the $1=0,1$ partial waves and with oniy the Coulomb potential for the higher waves. The theoretical correlation functions were averaged over the same directions as the experimental bins. Since the theoretical calculation does not include the dynamics necessary to predict the absolute probabllity of twoparticle to one-particle events, the theoretical curves were multiplied 
by a different factor for each energy gate to match the experinencal correlation function.

At high proton emission energies, both the longitudinal and transverse correlations are observed to exhibit strong enhaicements of approximately equal strength (see Fig. la). The similarity of the correlation in the two directions indicates a spherical emission source. The calculation shown In Fig. Ia corresponds to the correlation expected from a spherical source of negligible lifetime and compound nucleus dimensions, $R_{T}=R_{L}=4 \mathrm{fm}$. (Note that equivalent sharp sphere radii are $\sqrt{5 / 2}$ larger than the radil used in Eq. (2).] More surprising is the result geen in Fig. Ib that, although the correlation is weaker for low proton emission energies, no obvious directional dependence is observed. The weak angle-1ntegrated correlations observed for low-energy protons have previously been suggested to result from extended emission times, as from the later stages of an evaporation process. Calculated correlations are shown for an evaporative-type source with a transverse radius of $4 \mathrm{fin}$ and a longitudinal dimension of $20 \mathrm{fm}$. Although the calculated longitudinal correlation is in reasonable agreement with the experimental result, the theoretical transverse correlation is Pauli suppressed, due to the short transverse dimension, in contrast to the observed result. It is seen that a beter description of the low-energy proton result is obtained by the assumption of a spherlcal source of $6.5 \mathrm{fm}$ radius.

The difference between the calculated longttudinal and transverse correlations can also be decreased by increasing the longitudinal dimension (or, equivalently, increasing the lifetime) whlle keeping the transverse size fixed. This is 1llustrated in Fig. 2 where the calculated correlation for both directions at $q=25 \mathrm{MeV} / \mathrm{c}$ (where the two correlations differ stiongly) is shown as a function of the longitudinal size. 
It is seen that the two correlations again become simtlar for very large longitudinal radit, that is, for long lifetimes, but that the correlitinn Itself becomes very weak due to the tncreased volume, contrary to observation (see Fig. Ib).

The energy dependence of the proton emission source shape is shown in more detail in Fig. 3 for proton palr total kinetic energles ranging from $105 \mathrm{MeV}$ down to $55 \mathrm{MeV}$. The source radil were extracted by fitting the measured longitudinal and transverse correlations to the theoretical correlations. As noted in the discussion of Fig. I, although the highenergy protons appear to be emitted on a short timescale from a volume consistent with the combined system, the lower energy protons appear to be emitted from an extended spherical volume of up to twice the compound nucleus radius, rather than belng emitted over an extended period of time from the compound nucleus. The trend expected for the case of evaporative trmpound nucleus emission is also indicated in Fig. 3. In this case the transverse radius would remain equal to the compound nucleus radius $\left(R_{T}=4 \mathrm{fm}\right)$, while the longitudinal radius will appear to increase by the emission lifetime $\tau, R_{L}=\left(R_{T}^{2}+v_{K}^{2} \tau^{2}\right)$. It should be pointed out that the lowest proton energies considered here are still considerably above the Coulomb barrier, where a long evaporation timescale would clearly be expected. However, due to the experimental uncertainties and the weakness of the observed correlation at the lowest proton-pair kinetic energy of $55 \mathrm{MeV}$, the loose upper bound on the longitudinal radius allows that the emission source may in fact be elongated due to the emission timescale, although the large transverse radius does require a large spatial extent (see Fig. 3).

In conclusion, the observed directional dependence of the protonproton correlations indicates that the lower energy protons are enitted 
from an extended source considerably larger than the compound nucleus rather than from the compound system over an extended period of $t$ ime. At slightly lower bombarding energies, a similar diffuse emission source has also been inferred from the observation of low light-particle Coulomb energies, which could not be explained by deformation effects. 9 It is of interest that the temperatures expected to be attained for the present system are in the region of the nuclear I1quid-gas phase transition, and hence an increased eialssion volume would be expected.

\section{References}

\#This work was supported by the National Sclence Foundation under grant PHY-8608418 (University of Tennessee) and under grant PHY-8611210 (Michigan State University) and by the U.S. Department of Energy under contract DE-ACO5-840R21400 wath Martin Martetta Energy Systems, Inc. (ORNL).

tPresent address: Department of Physics, Oregon State University, Corvallis, Oregon 97331.

SPresent address: Institut fur Kernphysik, Johan-Wolfgang-Goethe Universitat, 6000 Frankfurt, Federal Republic of Germany.

1. W. G. Lynch et al., Phys. Rev. Lett. 51, 1850 (1983).

2. C. B. Chitwood et al., Phys. Lett. 172B, 27 (1986).

3. J. Pochodzalla et al., Phys. Lett. 174B, 36 (1986).

4. J. Pochodzalla et al., Phys. Rev. C 35, 1695 (1987).

5. Z. Chen et al., Nuc1. Phys. A473, 564 (1987).

6. David H. Boal and Hubert DeGuise, Phys. Rev. Lett. 57, 2901 (1986).

7. Scott Pratt and M. B. Tsang, Phys. Rev. C 36, 2390 (1987).

8. Steven E. Koonin, Phys. Lett. 70B, 43 (1977).

9. R. Lacey et al., Phys. Lett. 191B, 253 (1987). 
Figure Captions

Fig. 1. Proton-proton correlations for the $32 \mathrm{~S}+\mathrm{Ag}$ reaction at $715-\mathrm{MeV}$ incident energy. The correlation is shown for relative momenta in the longitudinal (filled circles) and transverse (open clrcles) direction relative to the direction of the total momentum of the proton pair. The correlations are shown for total laboracory kinetic energy of the proton pair of (a) $90<\mathrm{E}_{\mathrm{pP}}<100 \mathrm{MeV}$ together with the calculated correlation for $R_{T}=R_{L}=4 \mathrm{fm}$ and (b) $60<E_{P P}<70 \mathrm{MeV}$ together with calculations for $R_{T}=R_{L}=6.5 f_{m}$ (solid line) and $R_{T}=4 f_{m}, R_{L}=20 f m$ (longltudinal correlation: dashed line; transverse correlation: dot-dashed line). Errors reflect statistical uncertainty only.

F1g. 2. Longltudinal radius dependence of the longitudinal and transverse proton-proton correlations at a relative momentum of $25 \mathrm{MeV} / \mathrm{c}$ for a source with a transverse radius of $4 \mathrm{fm}$.

F1g. 3. Longltudinal and transverse rad11 obtalned by fitting the calculated correlations to the experimental longltudinal and transverse two-proton correlations for the ${ }^{32} \mathrm{~S}+\mathrm{Ag}$ reaction at $715 \mathrm{MeV}$. Results are shown for proton-palr kinetic energles ranging from 55 to $105 \mathrm{MeV}$. The error bars are conservative estimates obtalned by independent variations which double the $\chi^{2}$, which varled from 1.2 to 5.6. At $55 \mathrm{MeV}$ the upper bounds on the rad11 are essentially open. Note: the

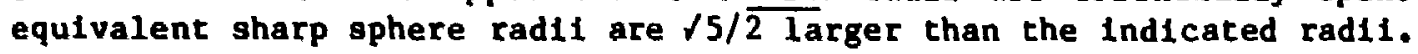




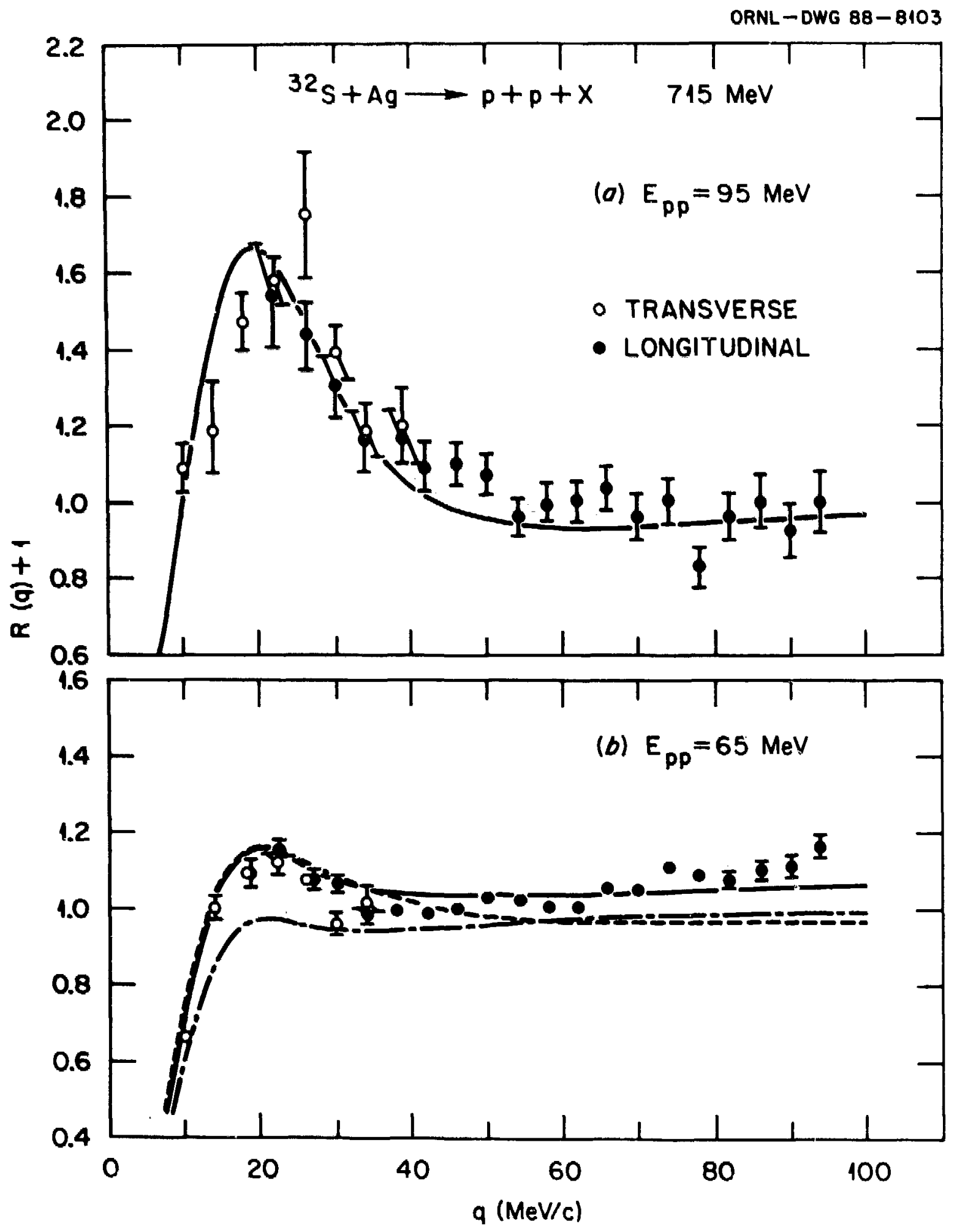




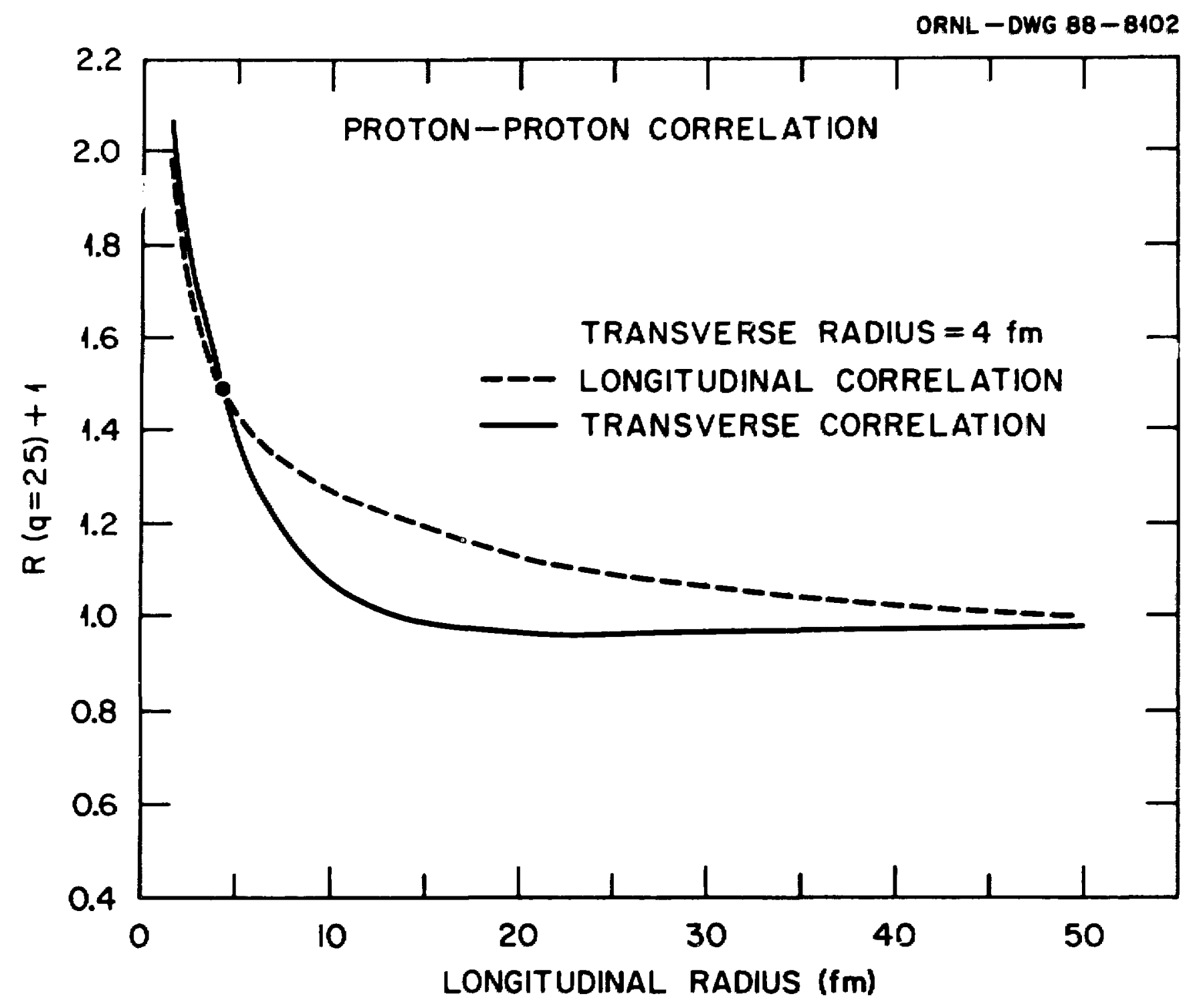




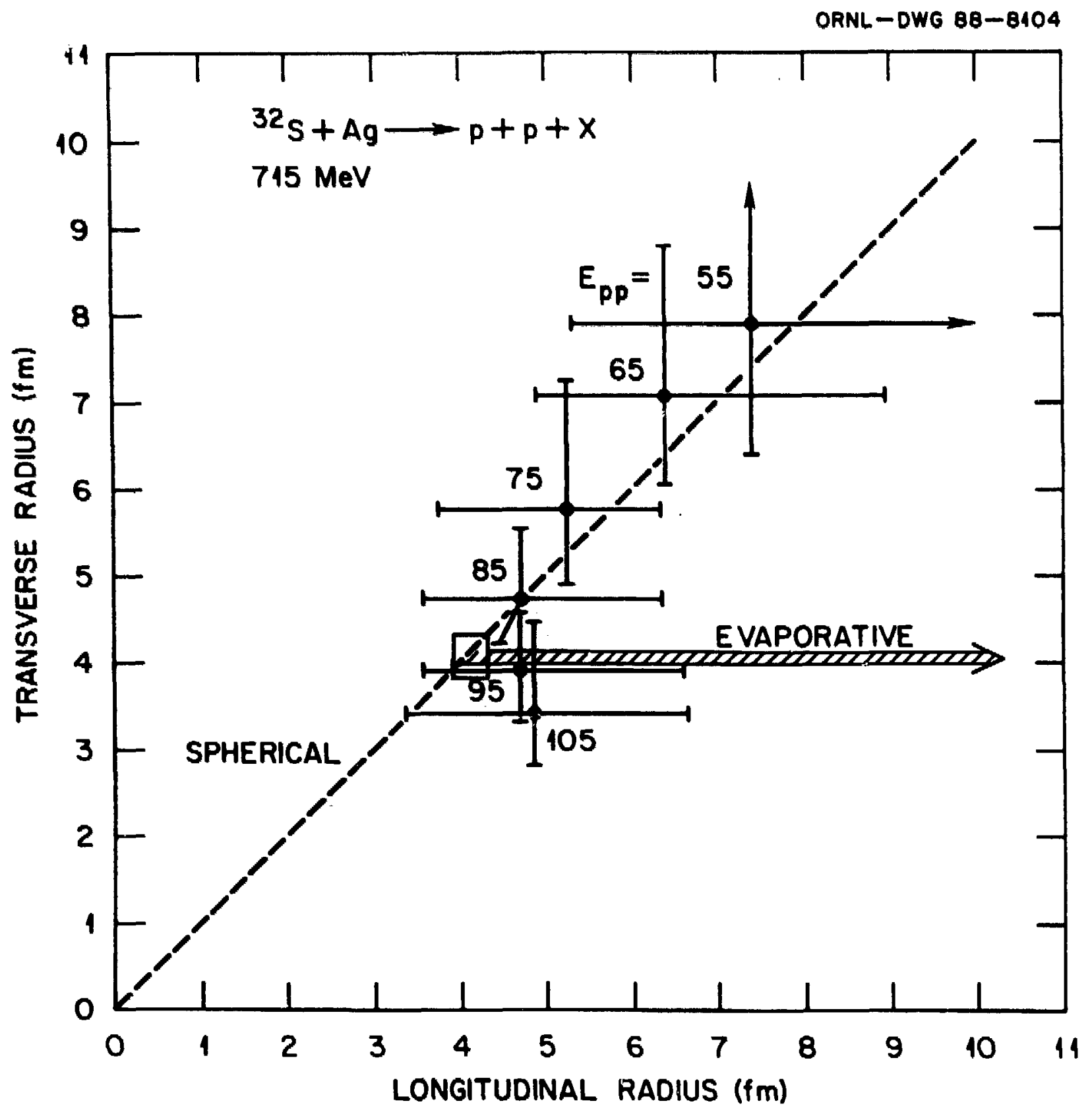




\section{DISCLAIMER}

This report was prepared as an account of work sponsored by an agency of the United States Government. Neither the United States Government nor any agency thereof, nor any of their employees, nakes any warranty, express or implied, or assumes any legal liability or responsibility for the accuracy, completeness, or usefulness of any information, apparatus, product, or process disclosed, or represents that its use would not infringe privately uwned rights. Reference herein to any specific commercial product, process, or service by trade name, trademark, manufacturer, or otherwise does not necessarily constitute or imply its endorsement, recommendation, or favoring by the United States Government or any agency thereof. The views and opinions of authors expressed herein do not necessarily state or reflect those of the United States Government or any sgency thereof. 